\title{
Questes
}

Revue pluridisciplinaire d'études médiévales

\section{"Teste David cum sibylla » : quelques autorités prophétiques citées pour annoncer la fin du monde}

Julien Abed

\section{(2) OpenEdition}

\section{Journals}

Édition électronique

URL : http://journals.openedition.org/questes/1594

DOI : 10.4000/questes.1594

ISSN : 2109-9472

Éditeur

Les Amis de Questes

\section{Édition imprimée}

Date de publication : 15 janvier 2006

Pagination : 55-62

ISSN : 2102-7188

\section{Référence électronique}

Julien Abed, « «Teste David cum sibylla » : quelques autorités prophétiques citées pour annoncer la fin du monde », Questes [En ligne], 8| 2006, mis en ligne le 01 janvier 2014, consulté le 01 mai 2019. URL : http://journals.openedition.org/questes/1594 ; DOI : 10.4000/questes.1594

Ce document a été généré automatiquement le 1 mai 2019.

(c) Association des amis de «Questes » 


\title{
«Teste David cum sibylla » : quelques autorités prophétiques citées pour annoncer la fin du monde
}

\author{
Julien Abed
}

1 Comment devenir un Maître de Vérité, une bouche qui ne soit pas accusée d'affabuler mais au contraire digne d'être crue ? Je vais m'intéresser ici aux noms propres qui sont utilisés par les poètes médiévaux pour cautionner les annonces réitérées de la fin du monde, la venue des temps derniers ou de l'Antéchrist. Il existe, dans le cas des autorités prophétiques que l'on cite à l'oral ou à l'écrit, un lien étroit entre la notion d'autorité et la figure de la métonymie, qui consiste à employer un mot pour un autre (ici, désigner le contenu d'un texte par le nom de son auteur supposé). La notion d'autorité a ici un effet psychologique: le charisme prophétique dont on revêt un simple nom engendre la soumission, voire la peur, et joue sur la crédulité des lecteurs et des auditeurs.

Ainsi, dans les très nombreux textes qui évoquent la fin du temps, le nom d'un prophète garantit l'efficacité du discours. On peut évoquer la formule liturgique bien connue :

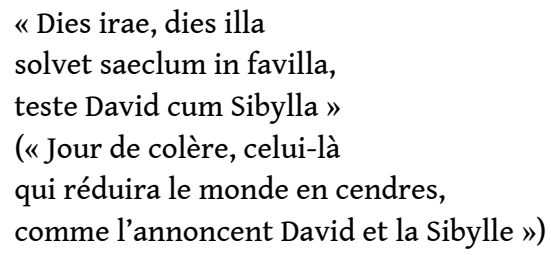

Le Moyen Âge possède un très grand nombre de textes qui parlent de la fin du monde. Des textes canoniques, d'abord, qui annoncent par le biais de l'énumération des cataclysmes la venue du Jour de Yahvé, et qui sont contenus dans l'Ancien testament (Isaïe, Daniel) ; puis un grand nombre de textes apocryphes, témoins de l'importante pénétration des idées eschatologiques dans le judaïsme tardif et le christianisme primitif, dans cette Antiquité finissante où le genre de l'apocalypse fait florès ${ }^{1}$. Ces derniers textes témoignent de l'interpénétration des scénarios de la fin du monde, car on a tendance à attribuer les annonces et les visions à des personnages religieux de premier plan : Esdras, Enoch, Abraham, Baruch, Pierre, Jean, Thomas, etc. 
Ce mécanisme assez naturel d'attribution abusive se poursuit et se développe au Moyen Âge, mais, à côté des figures vraiment prophétiques, l'époque médiévale a utilisé le cadre de la fiction pour en inventer d'autres, comme Merlin, ou a essayé d'élire des figures de l'Antiquité. Virgile, par exemple, devient une sorte de mage, de prophète, par la lecture chrétienne que l'on fait de sa quatrième Eglogue, et la sibylle, qu'il a décrite dans l'Énéide, connaît une destinée plus glorieuse encore. La " contagion » atteint même ceux qui commentent les textes apocalyptiques mais qui ne produisent pas eux-mêmes des textes visionnaires : Joachim de Flore, petit à petit, devient une autorité prophétique, alors que lui-même ne s'est jamais défini comme prophète.

Les noms fonctionnent donc comme des étiquettes auxquelles l'homme du Moyen Âge associe des contenus, des styles et, surtout, la capacité à prédire l'avenir. L'autorité prophétique possède en elle-même un charisme, justifié ou non, qui lui assure un crédit durable :

« Dans [un] contexte où il est impossible de distinguer ante eventum le vrai prophète des imposteurs, il est particulièrement important de donner d'emblée à la prédiction une aura de crédibilité. D'où les nombreuses auctoritates qui prêtent, nolens volens, leur nom à des traités de toutes sortes : Somniale Danielis ou Josephi, Lunaires d'Abel, de Salomon, Propheties d'Ezéchiel ou de Merlin, autant de tentatives de placer un contenu souvent simplet, païen ou aléatoire sous le patronage d'une figure tutélaire savante ou biblique. $»^{2}$

Cette multitude d'auctoritates (ou pseudo-auctoritates) constitue une sorte de personnel prophétique toujours prêt à l'emploi, que les écrivains utilisent comme cautions afin de garantir l'efficacité de leurs discours. Les noms des prophètes valent comme arguments, et fondent une rhétorique prophétique qui ne s'embarrasse pas des invraisemblances. Il y a, dans les textes prophétiques, une dissociation profonde entre la parole, lisible ou audible, et l'énonciateur à qui on les attribue. Pour formuler un peu grossièrement l'hypothèse, tout se passe comme si la divination médiévale ne se posait plus la question de l'origine de la parole, mais seulement celle de son autorisation.

7 Les modalités de la citation des noms prophétiques peut faire l'objet d'une étude stylistique : évidemment, les poètes ont d'abord recours à une citation de type formulaire, qui privilégie la sonorité des noms des prophètes (pour la rime), le rythme (recours fréquent à deux ou trois noms, plus rarement quatre), ou l'univers de référence (pour donner un arrière-plan historique).

8 Dans la formule liturgique qui sert de titre à cet article, David est mis aux côtés de la sibylle pour annoncer le jour de colère qui sera le point de départ du jugement de Dieu. Il n'y a visiblement pas de logique "auctoriale" au sens moderne: le nom et le texte suggéré par le nom sont indépendants, car la mentalité médiévale ne raisonne pas en termes d'origine (qui a écrit le texte ? qui a parlé ?) mais en termes de garant, d'autorité (qui autorise l'utilisation de cette parole?).

9 Je vais illustrer ces propos avec l'exemple d'un poème très connu et très apprécié au Moyen Âge, les « Quinze signes du jugement dernier ». Les nombreux manuscrits qui l'ont conservé prouvent à la fois sa popularité et son caractère mouvant ${ }^{3}$ : d'un manuscrit à l'autre, sa forme change (versification, forme strophique ou linéaire, prologue ou non), et l'attribution des visions varie.

10 Ainsi, dans le texte du poème tel qu'il est contenu dans un manuscrit appartenant à $M$. von Steiger à Berne, quatre Pères de l'Eglise, en fait les quatre grands docteurs latins de 
l'Eglise depuis le $\mathrm{VIII}^{\mathrm{e}}$ siècle, se donnent la main pour cautionner les apparitions des quinze signes :

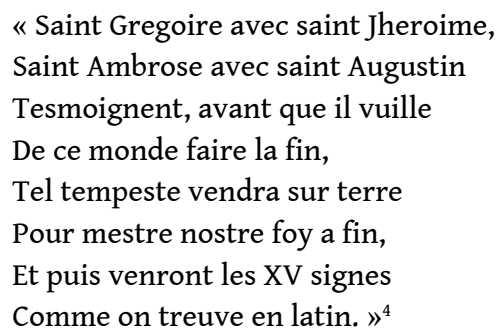

11 Mais dans le ms BNF fr 15212, la vision, rédigée en prose, n'est attribuée qu’à un seul prophète :

«Sains Jheromes dist que .XV. signe avenront devant le jour du jugement. ${ }^{5}$

Cette attribution est conforme à ce qu'écrit Jacques de Voragine au début de la Légende dorée, lorsqu'il confronte cette liste de quinze signes venue de saint Jérôme avec une autre liste de cinq signes venant de saint Luc. La question ne s'arrête pas là : c'est à la sibylle d'Erythrée, on le sait, que revient, dans la tradition augustinienne, le devoir d'annoncer le jour du Jugement ${ }^{6}$. Il en va ainsi dans les processions des prophètes, telles que nous les ont conservées quatre manuscrits médiévaux qui reposent, comme l'a montré Marius Sepet ${ }^{7}$, sur un sermon attribué à saint Augustin ${ }^{8}$.

Le poème, tel qu'il se trouve dans le BNF fr 837, commence comme un sermon: le prédicateur appelle l'attention du public («Oiez trestuit communement/ Dont Nostre Sires nous reprent $»^{9}$ ) et décrit la réalité avec un présent de vérité générale. Il se plaint, selon une topique bien connue, que l'homme en général aime mieux écouter l'histoire de Roland que celle de la Passion du Christ, et continue en annonçant l'avenir (« Ahii ! chetif, je morrons nous $! »^{10}$ ).

« Nous fesons trestuit que dolent:

nous en avrons grief jugement

quant icest siecle fenira

et Diex aus bons joie donra. ${ }^{11}$

Vient enfin la liste des garants :

"Quar quant li siecles fenira,

Nostre Sires signes fera,

Par quise jors voudra moustrer

Signes qui font a redouter!

Ce nous reconte Geremies,

Zorobabel et Ysaÿes,

De Babiloine Danïel,

Et si l'aferme Ezechïel,

Noé, Asmon et Moÿsés

Et li autre prophete après. $»^{12}$

Dans cette énumération, le nom est garant de la vérité de ce qui sera annoncé. L'autorité du prophète fonde le climat dans lequel se déroule la récitation ou la lecture, climat à la fois moral et intellectuel, qui correspond à l'image qu'on se fait de ceux qui récitent les signes avant-coureurs de la fin du monde, et de celui, dont on a toutes les raisons de penser qu'il est sérieux, qui rapporte ces propos. Les noms des autorités prophétiques permettent le bon fonctionnement du texte et engendrent la peur. Ce n'est qu'après que le «je » du prédicateur peut s'affirmer avec sérénité :

«Qui veut oïr la merveille

Drece son chief, si me regrt : 
Je li dirai ja de quel part

Vendra la grant mesaventure

Qui passera toute mesure. $»^{13}$

\section{huitième signe (la mer s'élèvera jusqu'au ciel, puis reviendra dans son lit), le poète cite un} garant prophétique tout désigné :

"Se cil ne ment qui ce nous dist :

C'est Moÿsés, qui ce escrist. ${ }^{14}$

L'auditeur est ici renvoyé à la Sainte Écriture, qui fonde tout le poème : si une telle chose a pu se passer dans les temps bibliques, alors il est vraisemblable qu'en des temps aussi troublés que ceux de la fin du monde, de tels prodiges se répètent. La rime "dist/ escrist » exprime bien le mouvement qui va du sermon à la grande autorité livresque. Le nom de Moïse est donc la caution dont se sert le poète pour affirmer la vérité de son propos (« Por voir, seignor, le vous afi »).

Pour le neuvième signe (tous les fleuves parleront), c'est un Père de l'Église qui est appelé :

«S'en trai a garant Augustin,

Qui de cest siecle dist la fin. $»^{15}$

Pour le dixième (le ciel et la terre se fendront et les diables sortiront de l'enfer),

«Ce nous afferme sainz Giroimes

Et li nobles clers sainz Grigoires. " ${ }^{16}$

Et un peu plus tard,

« Tuit en istront fors li deable :

Sainz Pols le dist, ce n'est pas fable. ${ }^{17}$

Pour le treizième, c'est le Livre de Job qui est convoqué :

«Ce ert signes de grant dolor.

De cestui dist Job en son livre

Que ja ne se verront delivre

Trestuit icil qui donc seront,

Més a Dieu merci crieront. $»^{18}$

Pour l'évocation finale du Jugement, le poète prend l'Évangile comme caution de ses dires :

« De l'Evangile oiez les dis. $»^{19}$

Le poète des Quinze signes du Jugement exploite la crédulité de son auditoire par des peintures étranges de la fin des temps. En recherchant les prodiges les plus dramatiques, il se réfère à plusieurs autorités prophétiques qui lui permettent d'accentuer la nature exceptionnelle des signes qu'il évoque. Or, il y a un apparent paradoxe à «traire a garant» autant de noms glorieux. L'écriture visionnaire devrait se passer d'autorité: reposant sur une vision, un songe, elle devrait en quelque sorte se suffire à elle-même, par son caractère visionnaire. Or, c'est tout le contraire qui se produit: la divination médiévale ne peut se passer d'autorités. Il lui faut toujours fonder les annonces inouïes dans une parole stable qui en garantisse la vérité et la crédibilité. Le nom du prophète porte témoignage et, par sa stature plus ancienne et plus imposante, il permet au poète d'exprimer l'inexprimable, et de décrire l'inconnu. On va donc à l'encontre d'une logique véritablement prophétique qui multiplierait les hypotyposes ou les apostrophes violentes. Le lien, présent dans le paganisme et le judaïsme, entre la poésie et la révélation du sacré, n'est plus possible dans les temps du christianisme : le poète ne peut se faire vates, car il serait pris pour un affabulateur, qui invente des fictions et orne le langage de façon trompeuse. "Teste David cum Sibylla » est la formule qui entend résoudre toutes les 
extravagances que le poète énonce ensuite. Par la mention d'autorités prophétiques, l'énonciateur s'autorise à accentuer la nature étrange des signes qu'il évoque.

En présentant le treizième signe relatif à la bataille des pierres, notre poète déclare que même ceux qui connaissent les langues, Japhet et Abraham, seraient incapables de dépeindre ce qui se passera à cette occasion :

«Li tresimes ert trop sauvages,

Quar cil qui sorent les langages

- Ce fu Japhet, li filz Noé,

Et Abraaham, li filz Tharé -

Ne porroient la moitié dire

Des granz dolors, de la grant ire

Que Nostre Sires mousterra

Quant icil signes avendra. $»^{20}$

C'est sans doute une habile transposition du «topos de l'ineffable» tel que Curtius l'a décrit : l'écrivain qui veut faire l'éloge d'une personne «accumule les noms d'écrivains célèbres qui ne seraient pas à la hauteur de la tâche $»^{21}$. Le poète assure ici que les prophètes antiques eux-mêmes pourraient décrire bien peu de choses parmi les faits extraordinaires qu'ils auraient à citer.

Par définition, ce qui est dit est invisible, indicible, ineffable; cela dépasse la parole seulement humaine, d'où la nécessité d'un garant efficace, d'une stature plus grande, plus ancienne, plus vieille. Comment la monstration des signes du Jugement peut-elle se faire? Comment, par des termes connus, décrire l'inconnu, comment exprimer l'inexprimable? Le recours aux autorités permet de contourner le problème des adynata et des impossibilia.

L'annonce des fins dernières se fonde sur la recherche d'autorités bien plus qu'elle ne sert l'invention poétique. Sans doute est-il vain de vouloir trouver une esthétique à l'œuvre dans ce type de texte prophétique, même versifié : c'est l'énonciation, en tant qu'elle repose sur un tissu d'autorités, et non le style, qui fait du vers prophétique le médium de la vérité.

\section{NOTES}

1. Seuls témoins de cet intense bouillonnement prophétique dans le texte biblique canonique : Matthieu XXV et l'Apocalypse de Jean.

2. Richard Trachsler, Préface à Moult oscure parleüre, Études sur la Prophétie médiévale recueillies par Richard Trachsler, avec la collaboration de Julien Abed et David Expert, volume publié avec le concours de l'UMR 8092 dirigée par Jacqueline Cerquiglini-Toulet et l'Institut Universitaire de France, Paris, Presses de l'université de Paris-Sorbonne, à paraitre en 2006.

3. C'est le texte qui suit par exemple le texte du jeu d'Adam et dont l'édition de Paul Aebischer a décidé de faire suite à la pièce : voir Le Mystère d'Adam (Ordo representationis Ade), éd. Paul AEBISCHER, Paris, Droz, 1964 (édition du ms Tours, Bibl. mun. 927).

4. Reine mANTou, "Le thème des "Quinze signes du Jugement dernier" dans la tradition française ", Revue Belge de Philologie et d'Histoire, XLV (3), 1967, pp. 827-841, voir p. 835. 
5. Ibid., p. 841.

6. SAINT AUGUSTIN, De civitate dei, XVIII, 23 dans MIGNE, Patrologia latina, XLI, cc. 579-581.

7. Voir Marius SEPET, Les Prophètes du Christ. Étude sur les origines du théâtre au Moyen Âge, Genève, Slatkine Reprints, 1974 [Paris, 1878].

8. PSEUdo-Augustin, "Sermo de symbolo adversus judaeos, paganos et arianos ", dans Migne, Patrologia latina, XLII, cc. 1126-1127.

9. Reine MANTOU, «Les Quinze signes du Jugement dernier, poème du XII ${ }^{\mathrm{e}}$ siècle. Édition critique ", Mémoires et Publications de la Société des Sciences, des Arts et des Lettres du Hainaut, 80e volume, Mons, 1966, pp. 112-212.

10. Ibid., p. 153.

11. Ibid., p. 153.

12. Ibid., p. 154-155.

13. Ibid., p. 155-156.

14. Ibid., p. 165.

15. Ibid., p. 166.

16. Ibid., p. 167.

17. Ibid., p. 168.

18. Ibid., p. 174.

19. Ibid., p. 179.

20. Ibid., p. 173.

21. Voir Ernst Robert cuRTIUS, La littérature européenne et le Moyen Âge latin, trad. fr. J. Bréjoux, Paris, Presses Universitaires de France, coll. Agora, 1986 [1ère éd. fr. 1956], p. 266.

\section{INDEX}

Mots-clés : autorité, David, prophétie, poète, discours, apocalypse

Keywords : authority, David, prophecy, annoncement, end, poet, cataclysm, speech, apocalypse 